\title{
THE ROLE OF CONTRAST-ENHANCED ULTRASONOGRAPHY IN EARLY RHEUMATOID ARTHRITIS
}

\author{
Maria-Magdalena Tamas ${ }^{1}$, Nicolae Rednic ${ }^{2}$, Simon Siao-Pin ${ }^{1}$, Simona Rednic $^{1}$ \\ ${ }^{1}$ Rheumatology Department, Iuliu Hatieganu University of Medicine and Pharmacy, \\ Cluj-Napoca, Romania \\ ${ }^{2} 4^{\text {th }}$ Medical Department, Railway Hospital, Cluj-Napoca, Romania
}

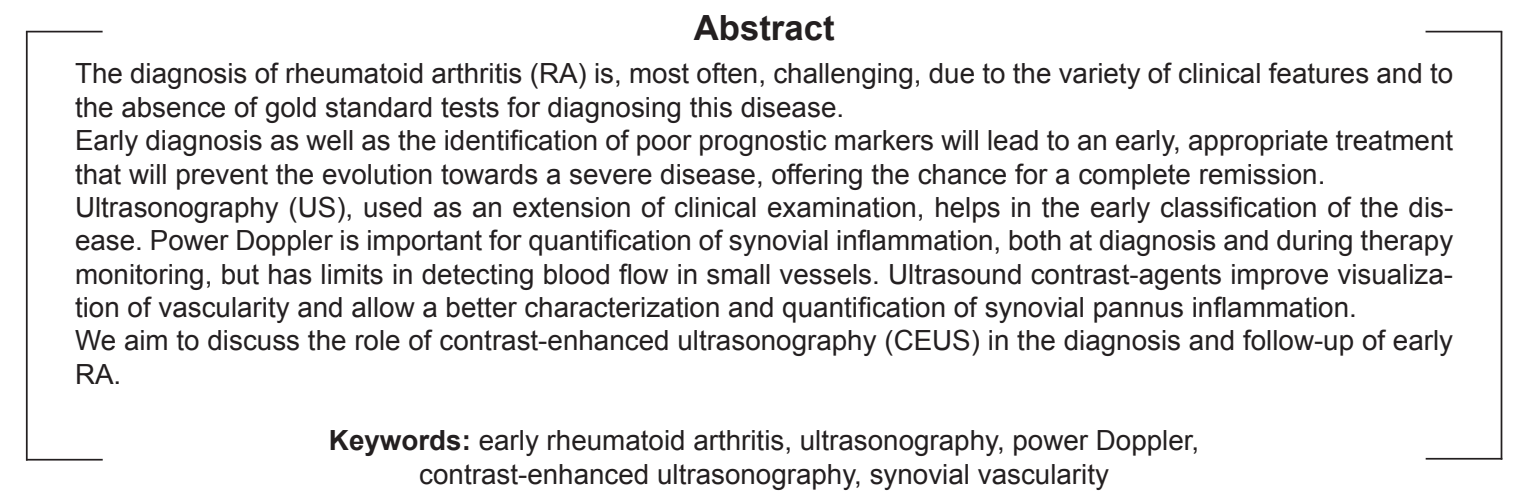

\section{INTRODUCTION}

Rheumatoid arthritis (RA) is a chronic multisystemic inflammatory arthritis, characterized by chronic synovial inflammation leading to progressive structural damage. If not treated, the synovial pannus infiltrate the subchondral bone and the disease progresses to joint space loss and significant disability. Early diagnosis is essential, as an early and aggressive treatment would delay the disease progression $(1,2)$.

The new ACR/EULAR criteria were developed to classify the disease in the early stages (3). Although clinical features are suggestive for this inflammatory arthritis, especially in the long-standing disease, different conditions may mimic RA in the early phases, sometimes leading to a delayed diagnosis.

During the last years, ultrasonography (US) became a useful imagistic tool for evaluating synovial hypertrophy, demonstrating a higher sensitivity in detecting synovitis, compared to clinical examina- tion (4-7). Moreover, power Doppler (PD) allows a good observation of pannus vascularization, indicating the degree of synovial inflammation. Studies demonstrate that the development of microvessels occurs early in the course of the disease, and that serologic markers for angiogenesis correlate with the disease activity and radiographic progression (810). Angiogenesis is related to joint destruction to a greater extent compared to pannus growth (11). Thus, imaging modalities like US and magnetic resonance imaging (MRI), by identifying the early vascular damage in RA, facilitate the diagnosis, especially in cases when clinical and laboratory features are insufficient (12).

Contrast-enhanced ultrasonography (CEUS) has the capacity of vizualising blood flow with low velocity, providing more information about synovial pannus vascularization compared to PD (13-17). To distinguish an active synovitis from joint fluid, blood

Correspondence address:

Maria-Magdalena Tamas, Department of Rheumatology, 2-4 Clinicilor Street, 400006 Cluj-Napoca, Romania

E-mail: mariam.tamas@gmail.com 
clots or fibrin using Grey-scale US (GSUS) may be difficult, due to the different degrees of echogenicity. CEUS has demonstrated the capability for differentiating an active pannus from fibrosis and necrosis and therefore the possibility to measure the thickness of the active synovial hypertrophy (16). Time-intensity curve (TIC) parameters calculation, using dedicated software, allow a better quantification of inflammation. Thus, CEUS represents an imaging technique of added value for evaluating both early and late stage RA (18).

\section{TECHNIQUE}

\section{Contrast agents}

Levovist, a first generation contrast agent, was used in several studies $(15,19-20)$. It contains air filled microbubbles, with a mean diameter of 2-3 $\mu \mathrm{m}$ stabilised by palmitic acid in a galactose based suspension, administred as a bolus or in a slow perfusion.

Sonovue is a second generation contrast agent containing sulfur hexafluoride microbubbles coated by a phospholipid capsule, as a lyophilisate powder. It is prepared in a liquid suspension by adding $5 \mathrm{ml}$ of saline solution to the powder before the administration. It is not soluble in blood and remains intravascular. The microbubbles have a mean diameter of $3 \mu \mathrm{m}$, similar to red blood cells.

After injection, microbubbles circulate in blood and reflect US more than the other tissues. The elimination half-time of Sonovue is 5-6 minutes and is exhaled through the lungs (21).

In contrast to the contrast medium used for MRI, US contrast agents remain intravasal, and will therefore reflect more accurately the abnormalities in the vascularized synovium (22). The post-contrast increase of echo intensity is an indicator of synovial vascularity, and therefore, of active synovial inflammation (23).

After bolus administration, the microbubbles become visible in the joint space and periarticular tissue in 20-30 seconds, the time being related to joints and vessels dimensions. The window of examination is usually 3-5 minutes, this time being longer only if the contrast agent is administered in microperfusion compared to bolus administration. Using bolus administration of the contrast agent, CEUS allows the examination of one or maximum two joints, because the microbubbles will be eliminated through lungs on approximately 5 minutes.

\section{Equipment}

US machines equipped with high frequency linear probes are used and a low mechanical index is necessary in order to minimize bubbles disruption. (22) Dedicated software both for CEUS technique as for measuring time-intensity curves parameters is needed.

\section{CEUS in early rheumatoid arthritis}

Early diagnosis is essential for the clinician, as an early and aggressive treatment would have a major impact on the evolution of the disease. Currently, RA progression is routinely assessed using conventional $\mathrm{X}$-Rays, while MRI and US enable the examination of soft tissues. MRI has proven the role in characterizing synovial inflammation, tendons, bursae and also bone erosions $(24,25)$. However, due to the high costs, this imaging method has a limited availability.

The role of US for assessing joint and tendon inflammation in RA, as well as bone damage, was already demonstrated (26-28). The research focused on CEUS has shown that this imaging method can improve the detection of these features in early RA (18).

\section{CEUS versus power Doppler}

PDUS is currently used for assessing the degree of synovial inflammation, being correlated with the disease activity, the radiographic progression and with a severe course of the disease in early RA (9, 29-31). Therefore, detection of pannus vascularization is essential both for diagnosis and for treatment monitoring in early RA patients. However, an important limit of PD would be the difficulty in assessing very small proliferated vessels in the synovial membrane, formed in the angiogenesis process. In contrast, CEUS is able to evaluate tissue perfusion independent from the flow speed (16). Published studies demonstrated that CEUS using a first generation contrast agent improved PD signal in the inflamed synovium, enabling detection of subclinical inflammation (19,32).

Moreover, due to microbubbles enhancement, CEUS avoids Doppler-specific artefact (16).

\section{Joints}

The capacity of CEUS to differentiate between an active synovial pannus and an old pannus or fibrous or necrotic tissue is useful both at diagnosis, and during the monitoring of the disease, allowing the optimal therapeutic intervention. (Fig. 1) 


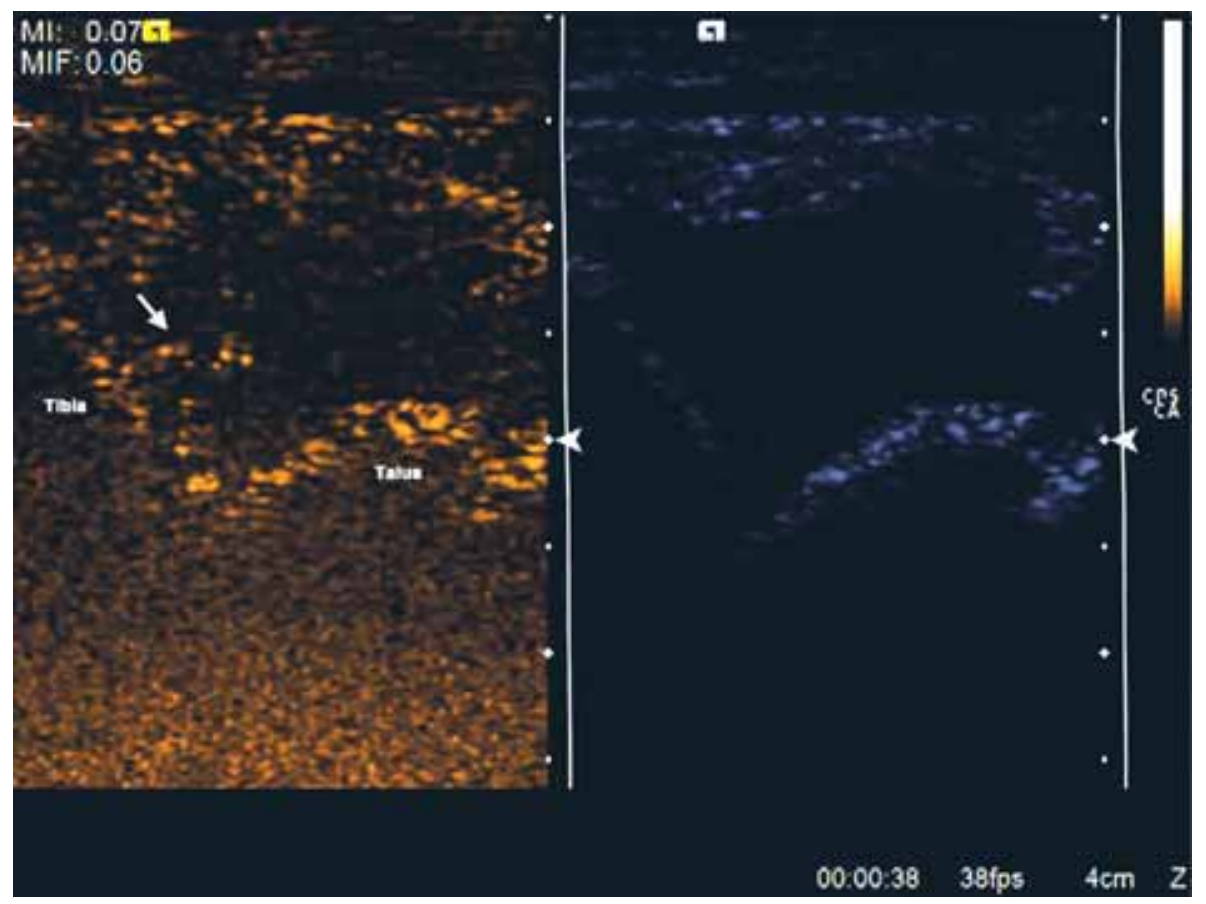

FIGURE 1. CEUS and GSUS of the left tibio-talar joint in a patient diagnosed with RA. CEUS image suggest an old pannus, with no enhancement, except a minimal accumulation of microbubbles (arrow). GSUS: grey-scale ultrasonography, CEUS: contrast-enhanced ultrasonography, $R A$ : rheumatoid arthritis

Persistent active synovitis is associated to poor prognosis and, consequently, to joint damage. Contrast agent administration allows a better evaluation of active synovitis compared to PDUS (16), thus increasing US sensitivity and improving the assessment of the disease activity. (Fig. 2 a, b)

In the study of Klauser et al, CEUS allowed differentiation of active synovitis from inactive synovial thickening in $97.3 \%$ compared to $60.1 \%$ of patients if only GS and PDUS were used. The thickness of the synovium was more accurately measured after contrast administration (16). Earlier studies demonstrated that synovial volumes determined using MRI were correlated to the disease inflammation, suggesting that synovial inflammation may be considered a predictor of the disease activity (33).

It has been demonstrated that subclinical disease is present in a significant proportion of patients with RA. Residual positive PD was found in $41 \%$ to $43 \%$ of patients considered in clinical remission $(34,35)$. CEUS enabled the detection of inflammation in $49 \%$ of clinically inactive joint and in $98 \%$ of moderate active joints, while PD in $8 \%$ and $52 \%$, respectively, demonstrating an improvement in the detection of blood flow in the hand small joints (15). These observations underline the importance of US examination also for the evaluation of joints less clinically involved.

As synovial hypertrophy is correlated with a more severe disease, its thickness measurement could be a useful parameter for treatment follow-up (16).

\section{Tendons}

The benefits of CEUS are reported not only when evaluating joints, but also for demonstrating tendon and bursa inflammation.

Tenosynovitis is a frequent finding in RA, responsible for pain, physical disability and for structural damage. Complications like tendon-ruptures may also occur. US is currently used for evaluating the extension of the disease at tendon level in RA, as these abnormalities are not visible using conventional X-Rays. Flexor digitorum tendons and wrist extensors are usually involved in RA, extensor ulnaris carpis being more frequently inflamed in the early stages of the disease (36). Regional perfusion within tendons and tendon sheats using CEUS was less studied and there is only a few data published in healthy individuals $(37,38)$ In a study based on experimental rabbit model, the authors have shown temporal changes in vascularity of Achille tendon injected with collagenase at various doses, while PD was less useful for assessment of neovascularization (39).

Klauser et al. demonstrated that CEUS was more sensitive compared to PD in identifying tendon vascularity in RA patients. The extent of vascularity was better evaluated using CEUS and was not correlated with tenosynovial thickening (40).

\section{Bone erosions}

Bone erosions represent the hallmark of RA and their early identification is important, as they are con- 


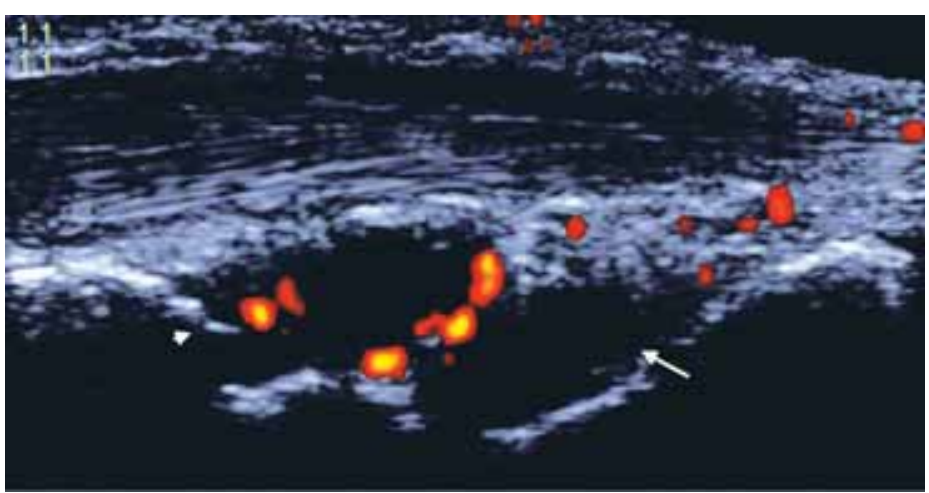

a

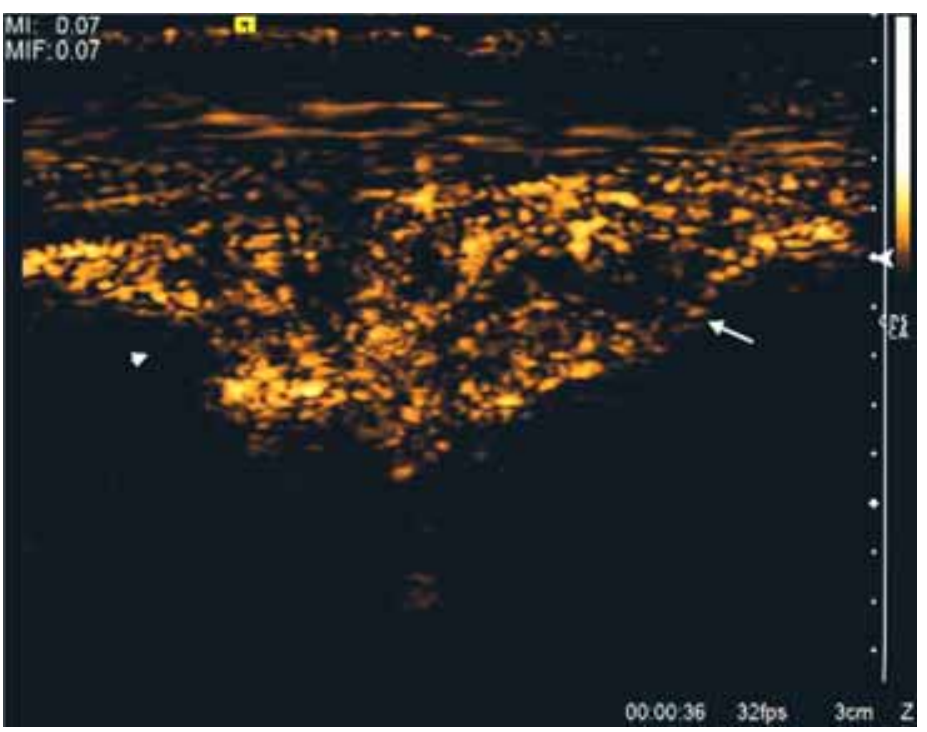

b

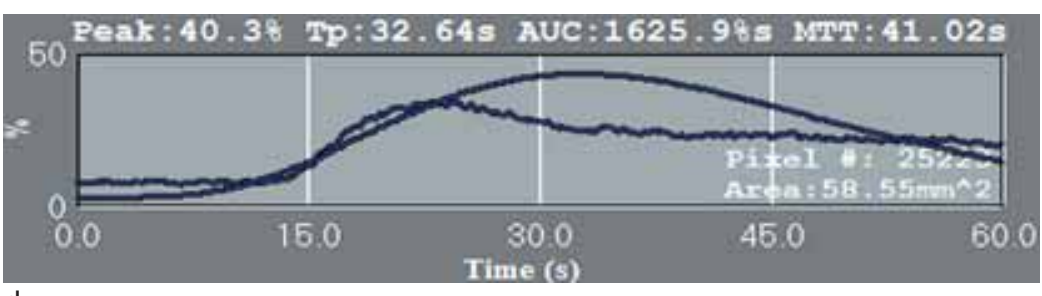

d

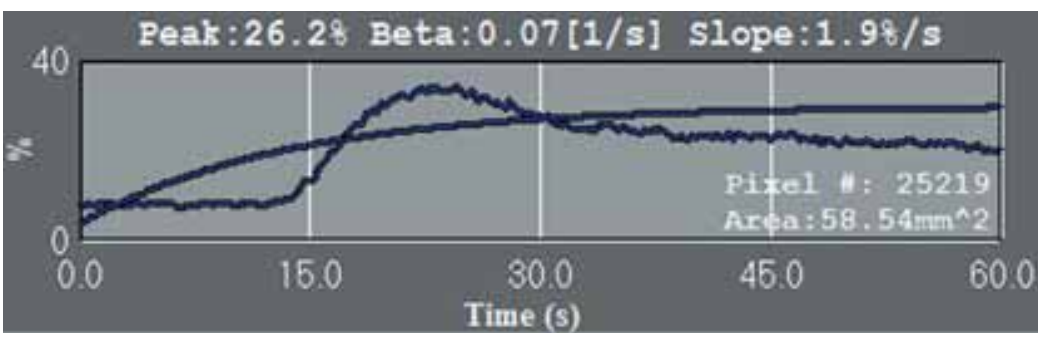

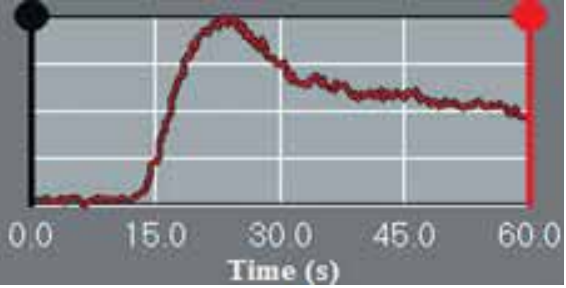

c

FIGURE 2. (a) PD, (b) CEUS, (c) TIC, (d) gamma, (e) exponential analysis PDUS, CEUS and TIC of the right wrist in a patient diagnosed with early active $R A$. CEUS indicate significant enhancement in the RC (arrowhead) and IC joints (arrow), although minimal PD is present. TIC show quantitative values of the calculated parameters, in Gamma and Exponential analysis.

PDUS: power Doppler ultrasonography, CEUS: contrast-enhanced ultrasonography, TIC: time-intensity curves, RC: radiocarpal, IC: intercarpal, $R A$ : rheumatoid arthritis

e

sidered a poor prognostic factor $(41,42)$. A significant percentage of patients develop bone erosions during the first year of the disease (43). Bone erosions first arise at the bare areas due to the lack of cartilage and consequent to synovial pannus invasion. Using MRI, early bone erosions may be identified in a significant percentage of patients during the first months of the disease (44). Due to the easy access of the US beam to the anatomical sites frequently involved in RA, US has been proved of value for rapid identification of bone erosions, including the early stages of the disease (45-49). CEUS may provide additional information related to bone erosion vascularization.

\section{CEUS versus MRI}

A good correlation between CEUS and MRI for the detection and characterisation of synovits was seen in various published studies $(19,32,50)$. 
In the study of Ohrndorf et al patients with RA were examined using US, CEUS and MRI before and after receiving anti TNF-alpha treatment (51). The results showed that CEUS expressed the highest correlation with MRI, demonstrating an increased sensitivity compared to GS and PD. In this study, both subjective and objective quantification, using the slope value of the time-intensity curves, correlated with MRI. The authors suggested that, due to its high correlation with MRI, CEUS examination of the clinically most affected joint, may reflect the disease activity (51).

\section{CEUS versus Histopathology}

Systemic autoimmunity precedes the development of clinical signs and symptoms of RA by several years (52) and is also present before the development of synovial inflammation in patients at risk for developing RA (53). Previous studies have shown that cell infiltration and the expression of inflammatory cytokines are similar in early and long standing RA $(54,55)$.

In early RA, the maximal lining thickness of the synovial membrane was demonstrated to be significantly thinner that in late stage RA. There are differences regarding the vascular changes in the synovium between RA and other inflammatory joint pathologies, in the early phases of the disease, with straight vessels in RA compared to a bushy and tortuous appearance of vessels in psoriatic arthritis and reactive arthritis (56). In early RA there is increased vascularity. However, it seems that no differences regarding macroscopic vascularity in early versus late disease were demonstrated $(55,57)$.

Few studies comparing CEUS abnormalities and histopathological aspects of the synovium in RA have been published. In the early phases of RA, CEUS better enabled the vascular flow when compared to PD or colour Doppler. (58)

Jiang Y. (2011) found correlations between the immunohistochemical markers of neovascularisation in the synovial pannus and TIC parameters (peak, area under the curve, and slope) (59).

In another study on animal models, CEUS was significantly correlated with synovial thickness of the synovium in knee antigen-induced arthritis. Synovial thickness measured using CEUS was better correlated with the pathology synovitis score in the chronic phase of inflammation compared to capsule thickness measured by US without contrast (60).

\section{Quantification of inflammation}

Quantification is needed to evaluate data objectively, to characterize inflammation in a specific area, to monitor the therapeutic response (61).

In this new era of biological therapy it would be ideal to have the possibility of measuring and quantifying the degree of synovial vascularity in RA.

CEUS examination allows synovial inflammation evaluation using semiquantitative scores and quantitative assessment by measuring the synovial membrane thickness or by analyzing the time-intensity curves.

\section{Subjective grading of contrast enhancement}

The semiquantitative scoring system for CEUS is reproducible and practical, but studies are still needed (23).

There is no consensus regarding the abnormalities that need to be scored: the intensity of enhancement, the quantity of microbubbles or the area covered with microbubbles.

There is published data documenting the role of subjective grading of CEUS in patients with RA (15). The authors quantified the degree of enhancement for evaluating the activity of synovial hypertrophy, providing a semiquantitative score, from inactive to moderately active and to highly active joint.

Klauser et al. studied the subjective versus the objective CEUS in RA patients. The results showed that both the intensity and the extent of vascularization were significantly higher when assessed with CEUS than with PDUS. No correlations between clinical activity and sonographic vascularity were found. The objective method correlated with PDUS grading. A significant correlation between the two modalities of CEUS examination was observed; however the authors suggests that objective measurement may be important for therapy follow-up (62).

\section{Objective measurement of contrast enhancement using TIC}

TIC parameters are descriptive and allow an objective quantification of vascularization in the analyzed anatomical area. (Fig. 2. c, d, e) Choosing the region of interest for calculating TIC may play an important role for an accurate assessment of synovial inflammation (23). There are several TIC parameters described - some of them being related to blood 

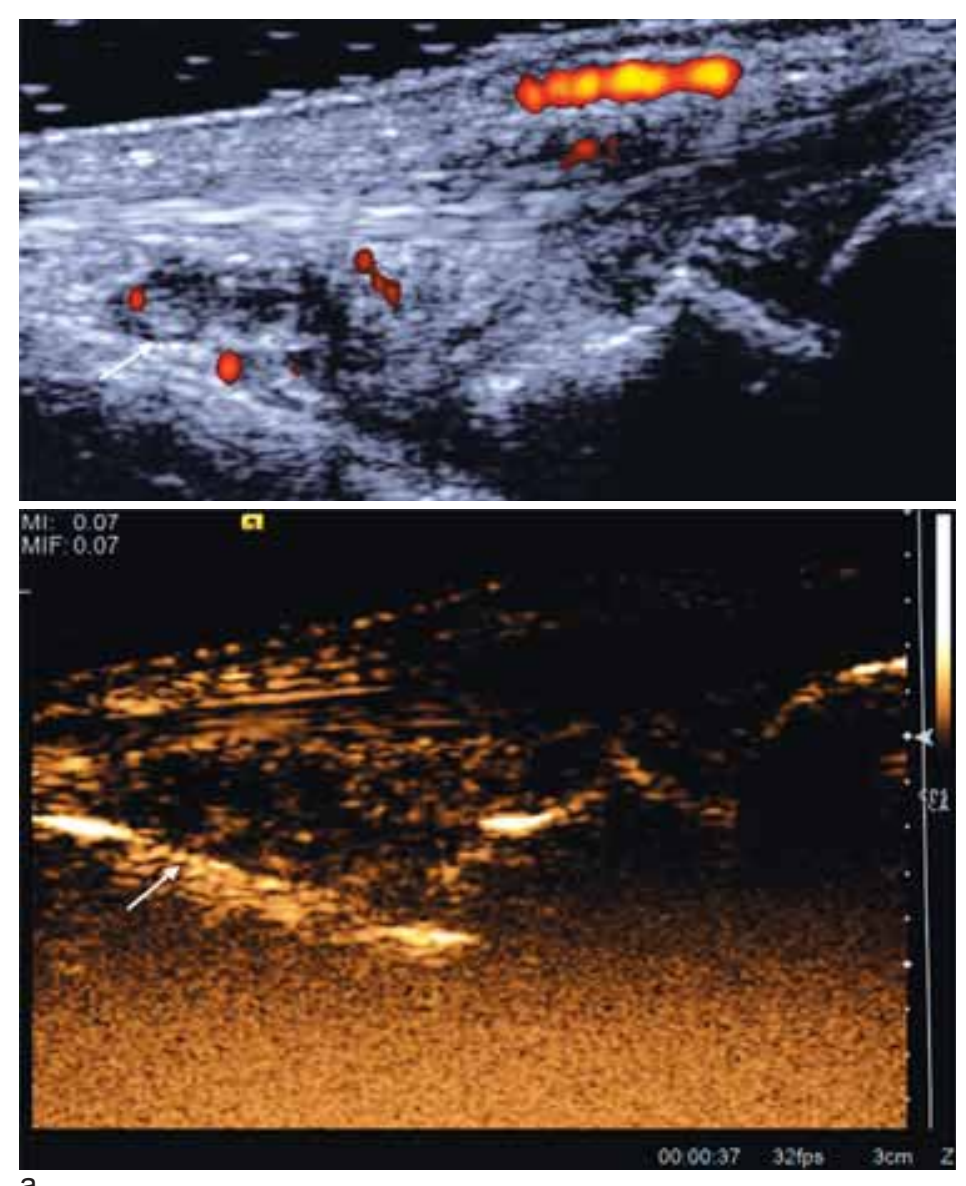

a
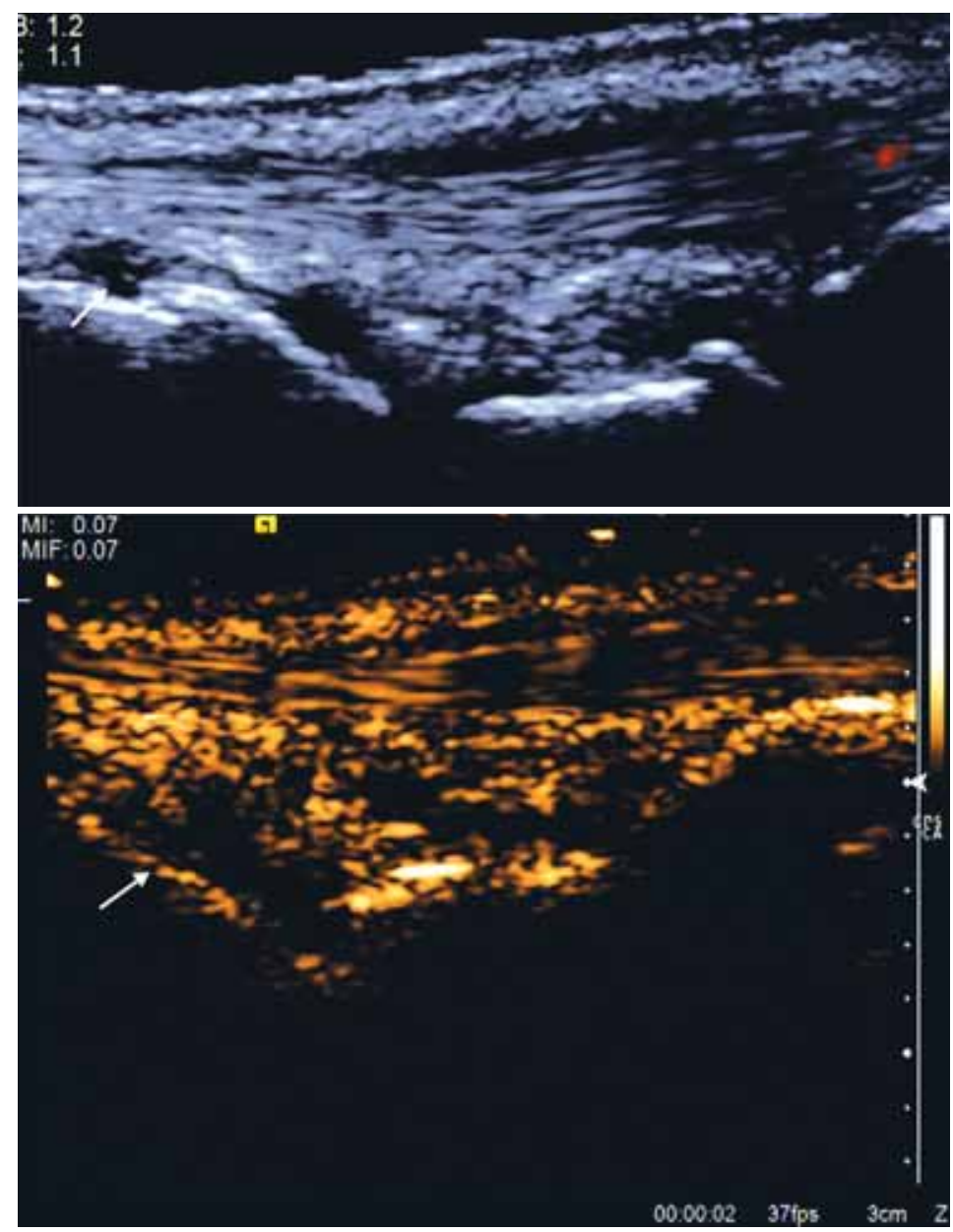

FIGURE 3. CEUS enhancement of the right IC joint (arrow) at baseline (a) and at 6 months follow-up (b) in a patient diagnosed with early $R A$, treated with conventional synthetic drugs. CEUS: contrast-enhanced ultrasonography, IC: intercarpal, RA: rheumatoid arthritis 
volume (peak intensity, area under the curve), while others related to blood flow (time-to-peak, slope of the ascending curve) (61). Studies on animal models demonstrated that TIC parameters correlated with the immunohistochemical findings in the inflamed joint tissue. Thus, TIC parameters would allow a true quantification of the disease activity (59).

Platzgummer showed a good correlation between different TIC parameters and PD, raising the question if CEUS is really needed in the daily clinical practice.

Although both subjective and objective methods are shown to be useful in demonstrating synovial inflammation, quantification using TIC would allow a better assessment of the disease activity and could be used to analyze the response to treatment $(59,62)$.

\section{Treatment monitoring}

To differentiate between a fibrotic pannus and an active synovitis is of help not only at diagnosis, but also in the treatment follow-up. The decrease in vascularity as well as the reduction of the inflamed tissue are important for establishing the response to treatment. (Fig. 3. a, b)

Less published data exists regarding the treatment monitoring in RA using CEUS. Salaffi et al demonstrated that oral/ intraarticular corticosteroids lead to a significant decrease of the area under the curve of contrast enhancement in patients with rheumatoid arthritis and knee synovitis (63).

In the study of Ohrndorf et al., the authors demonstrated that PD and CEUS were both sensitive methods for detecting synovial inflammation and useful for the anti-TNF alpha therapy monitoring (51).

In another study, both PD and quantitative CEUS (using the area under the time-intensity curve) al- lowed detection of synovial vascularity in finger joints in RA patients before and after corticosteroids treatment, but minor changes of vascularity were better evaluated using CEUS (64).

\section{Limits}

Limitations to the use of CEUS include the halftime of the contrast agent, and, as a consequence, the small number of joints that can be evaluated in one examination. The technique is microinvasive and exhibits increased costs, which possible limit its use. Prolonged examination and interpretation time represents another limitation of CEUS use.

The lack of sufficient data for CEUS in healthy joints and the absence of cut-off values for TIC parameters also limit its use for diagnosis purposes and for therapy monitoring.

Operator-dependency of US imaging is one of the major limitations that may influence the results and their interpretation.

\section{CONCLUSION}

In early RA, US should be used as the first-line imaging technique, both for diagnosis and follow-up. CEUS further allows a more sensitive assessment of synovial vascularity, adding information on the disease activity. CEUS abnormalities with significant impact for rheumatologists, as well as their prognostic value, still remain to be determined.

\section{Acknowledgement}

This paper was published with financial support of European Social Fund, Human Resources Development Operational Programme 2007-2013, POSDRU grant no. 159/1.5/S/138776.

\section{REFERENCES}

1. Finckh A., Liang M.H., van Herckenrode C.M., et al. Longterm impact of early treatment on radiographic progression in rheumatoid arthritis: A meta-analysis. Arthritis Rheum 2006; 55(6):864-72

2. Kyburz D., Gabay C., Michel B.A., et al. The long-term impact of early treatment of rheumatoid arthritis on radiographic progression: a population-based cohort study. Rheumatology 2011:50(6):1106-1110

3. Aletaha D., Neogi T., Silman A.J., et al. 2010 Rheumatoid arthritis classification criteria: an American College of Rheumatology/ European League Against Rheumatism collaborative initiative. Arthritis Rheum. 2010 Sep;62(9):2569-81

4. Kane D., Balint P.V., Sturrock R.D. Ultrasonography is superior to clinical examination in the detection and localization of knee joint

effusion in rheumatoid arthritis. J Rheumatol 2003 May; 30(5):96671.

5. Wakefield R.J., Green M.J., Marzo-Ortega H., et al. Should oligoarthritis be reclassified? Ultrasound reveals a high prevalence of subclinical disease. Ann Rheum Dis 2004, 63(4):382-5.

6. Naredo E., Bonilla G., Gamero F., et al. Assessment of inflammatory activity in rheumatoid arthritis: a comparative study of clinical evaluation with grey scale and power Doppler ultrasonography. Ann Rheum Dis. 2005 Mar; 64(3):375-381.

7. Ten Cate, D.F. Role of ultrasonography in diagnosing early rheumatoid arthritis and remission of rheumatoid arthritis - a systematic review of the literature. Arthritis Res \& Ther, 2013, 15:R4. 
8. Walsh D.A. Angiogenesis and arthritis Rheumatology 1999; 38:103-112

9. Clavel G., Bessis N., Lemeiter D., et al. Angiogenesis markers (VEGF, soluble receptor of VEGF and angiopoietin-1) in very early arthritis and their association with inflammation and joint destruction. Clin Immunol 2007 Aug; 124(2):158-64.

10. Kawashiri S.Y., Kawakami A., Iwamoto N., et al. The power Doppler ultrasonography score from 24 synovial sites or 6 simplified synovial sites, including the metacarpophalangeal joints, reflects the clinical disease activity and level of serum biomarkers in patients with rheumatoid arthritis Rheumatology 2011 May; 50(5):962-5

11. Firestein G.S. Starving the synovium: angiogenesis and inflammation in rheumatoid arthritis. J Clin Invest 1999; 103(1):3-4

12. Backhaus M., Kamradt T., Sandrock D., et al. Arthritis of the finger joints: a comprehensive approach comparing conventional radiography, scintig- raphy, ultrasound, and contrast-enhanced magnetic resonance imaging. Arthritis Rheum 1999; 42(6):1232-45.

13. Carotti M., Filippucci E., Salaffi F. Power Doppler e mezzi di contrasto nello studio della membrana sinoviale reumatoide. Reumatismo 2002; 54:361-3.

14. Doria A.S., Kiss M.H., Lotito A.P., et al. Juvenile rheumatoid arthritis of the knee: evaluation with contrast-enhanced color Doppler ultrasound. Pediatr Radiol 2001; 31: 524-531

15. Klauser A.S., Frauscher F., Schirmer M., et al. The value of contrast-enhanced Color Doppler ultrasound in the detection of vascularization of finger joints in patients with rheumatoid arthritis. Arthritis Rheum 2002; 46(3):647-53.

16. Klauser A., Demharter J., De Marchi A., et al. Contrast enhanced gray-scale sonography in assessment of joint vascularity in rheumatoid arthritis: results from the IACUS study group. Eur Radiol 2005; 15:2404-10.

17. Klauser A., Frauscher F. Schirmer M. Value of contrast-enhanced power Doppler ultrasonography of the metacarpophalangeal joints on rheumatoid arthritis. Eur Radiol 2004; 14:545-6.

18. De Zordo T., Mlekusch S.P., Feuchtner G.M., et al. Value of contrast-enhanced ultrasound in rheumatoid arthritis. Eur J Radiol 2007; 64: 222-30

19. Szkudlarek M., Court-Payen M., Strandberg C., et al. Contrast-enhanced power Doppler ultrasonography of the metacarpophalangeal joints in rheumatoid arthritis. Eur Radiol 2003; 13:163-168

20. Terslev L., Torp-Pedersen S., Bang N., et al. Doppler ultrasound findings in healthy wrists and finger joints before and after use of two different contrast agents. Ann Rheum Dis 2005; 64:824-827.

21. Morel D.R., Schwieger I., Hohn L., et al. Human pharmacokinetics and safety evaluation of SonoVue, a new contrast agent for ultrasound imaging. Invest Radiol 2000 Jan; 35(1):80-85

22. Tang M.X., Mulvana H., Gauthier T., et al. Quantitative contrastenhanced ultrasound imaging: a review of sources of variability. Interface Focus 2011; 1(4):520-539

23. Platzgummer H., Schueller G., Grisar J., et al. Quantification of synovitis in Rheumatoid Arthritis: Do we really need quantitative measurement of contrast-enhanced ultrasound? Eur J Radiol 2009; 71:237-241.

24. Sugimoto H., Takeda A., Hyodoh K. Early-stage rheumatoid arthritis: prospective study of the effectiveness of MR imaging for diagnosis. Radiology 2000; 216:569-575

25. Boyesen M., Ostergaard M., Cimmino M.A., et al. MRI quantification of rheumatoid arthritis: current knowledge and future perspectives. Eur J Radiol 2009; 71(2):189-196

26. Grassi W., Tittarelli E., Pirani O., et al. Ultrasound examination of metacarpophalangeal joints in rheumatoid arthritis. Scand J Rheumatol 1993; 22:243-247.

27. Wakefield R.J., Gibbon W.W., Conaghan P.G., et al. The value of sonography in the detection of bone erosions in patients with rheumatoid arthritis. Arthritis Rheum 2000: 43:2762-70.

28. Schmidt W.A. Value of sonography in diagnosis of rheumatoid arthritis. Lancet 2001; 357:1056-1057.

29. Freeston J.E., Wakefield R.J., Conaghan P.G., et al. A diagnostic algorithm for persistence of very early inflammatory arthritis: the utility of power Doppler ultrasound when added to conventional assessment tools. Ann Rheum Dis 2010; 69(2):417-419.
30. Filer A., De Pablo P., Allen G., et al. Utility of ultrasound joint counts in the prediction of rheumatoid arthritis in patients with very early synovitis. Ann Rheum Dis 2011; 70(3):500-507.

31. Salaffi F., Ciapetti A., Gasparini S., et al. A clinical prediction rule combining routine assessment and power Doppler ultrasonography for predicting progression to rheumatoid arthritis from early-onset undifferentiated arthritis. Clin Exp Rheumatol 2010; 28(5):686-694.

32. Magarelli N., Guglielmi G., Di Matteo L., et al. Diagnostic utility of an echo-contrast agent in patients with synovitis using power Doppler ultrasound: a preliminary study with comparison to contrast-enhanced MRI. Eur Radiol 2001; 11:1039-1046.

33. Ostergaard M., Stoltenberg M., Lovgreen-Nielsen $P_{\text {., }}$ et al. Magnetic resonance imaging-determined synovial membrane and joint effusion volumes in rheumatoid arthritis and osteoarthritis: comparison with the macroscopic and microscopic appearance of the synovium. Arthritis Rheum 1997; 40(10):1856-1867.

34. Brown A.K., Quinn M.A., Karim Z., et al. Presence of significant synovitis in rheumatoid arthritis patients with disease-modifying antirheumatic drug-induced clinical remission: evidence from an imaging study may explain structural progression. Arthritis Rheum 2006; 54(12):3761-3773.

35. Scirè C.A., Montecucco C., Codullo V., et al. Ultrasonographic evaluation of joint involvement in early rheumatoid arthritis in clinical remission: power Doppler signal predicts short-term relapse. Rheumatology 2009; 48(9):1092-1097

36. Backhaus $\mathbf{M}$. Ultrasound and structural changes in inflammatory arthritis: synovitis and tenosynovitis. Ann N Y Acad Sci 2009, 1154:139-151

37. Morel M., Boutry N., Demondion X., et al. Normal anatomy of the heel entheses: anatomical and ultrasonographic study of their blood supply. Surg Radiol Anat 2005, 27:176-183.

38. Koenig M.J., Torp-Pedersen S., Holmich P., et al. Ultrasound Doppler of the Achilles tendon before and after injection of an ultrasound contrast agent: findings in asymptomatic subjects. Ultraschall Med 2007; 28:52-56.

39. Chang K.V., Wu C.H., Ding Y.H., et al. Application of ContrastEnhanced Sonography With Time-Intensity Curve Analysis to Explore Hypervascularity in Achilles Tendinopathy by Using a Rabbit Model. J Ultrasound Med 2012; 31:737-746.

40. Klauser A.S., Franz M., Arora R., et al. Detection of vascularity in wrist tenosynovitis: power doppler ultrasound compared with contrast-enhanced grey-scale ultrasound. Arthritis Res \& Ther 2010, 12:R209.

41. Van der Heijde D.M. Joint erosions and patients with ERA. $B r$ J Rheumatol 1995; 34(Suppl 2):74-78.

42. Mottonen T.T. Prediction of erosiveness and rate of development of new erosions in ERA. Ann Rheum Dis 1998; 47:648-653.

43. McQueen F.M., Stewart N., Crabbe J., et al. Magnetic resonance imaging of the wrist in early rheumatoid arthritis reveals a high prevalence of erosions at four months after symptom onset. Ann Rheum Dis 1998 Jun; 57(6):350-356.

44. McQueen F.M., Stewart N., Crabbed J., et al. Magnetic resonance imaging of the wrist in early rheumatoid arthritis reveals progression of erosions despite clinical improvement. Ann Rheum Dis 1999; 58(3):156-163.

45. Wakefield R.J., Gibbon W.W., Conaghan P.G., et al. The value of sonography in the detection of bone erosions in patients with rheumatoid arthritis: a comparison with conventional radiography Arthritis Rheum 2000; 43:2762-2770.

46. Grassi W., Filippucci E., Farina A., et al. Ultrasonography in the evaluation of bone erosions. Ann Rheum Dis 2001; 60: 98-103.

47. Magnani M., Salizzoni E., Mulè R., et al. Ultrasonography detection of early bone erosions in the metacarpophalangeal joints of patients with rheumatoid arthritis. Clin Exp Rheumatol 2004; 22:743-748

48. Hammer H.B., Haavardsholm E.A., Bøyesen P., et al. Bone erosions at the distal ulna detected by ultrasonography are associated with structural damage assessed by conventional radiography and MRI: a study of patients with recent onset rheumatoid arthritis. Rheumatology 2009; 48: 1530-1532.

49. Tămaş M.M., Filippucci E., Becciolini A., et al. Bone erosions in rheumatoid arthritis: ultrasound findings in the early stage of the disease. Rheumatology 2014 Jun; 53(6):1100-1107 
50. Song I.H., Althoff C.E., Hermann K.G. et al. Knee osteoarthritis efficacy of a new method of contrast-enhanced musculoskeletal ultrasonography in detection of synovitis in patients with knee osteoarthritis in comparison with magnetic resonance imaging. Ann Rheum Dis 2008;67:19-25.

51. Ohrndorf S., Hensch A., Naumann L., et al. Contrast-enhanced ultrasonography is more sensitive than grayscale and power Doppler ultrasonography compared to MRI in therapy monitoring of rheumatoid arthritis patients. Ultraschall Med 2011 Dec; 32 Suppl 2:E38-44.

52. Nielen M.M., van Schaardenburg D., Reesink H.W., et al. Specific autoantibodies precede the symptoms of rheumatoid arthritis: a study of serial measurements in blood donors. Arthritis Rheum 2004; 50:380-386.

53. van de Sande M.G., de Hair M.J., van der Leij C., et al. Different stages of rheumatoid arthritis: features of the synovium in the preclinical phase. Ann Rheum Dis 2011; 70:772-777.

54. Tak P.P., Smeets T.J., Daha M.R., et al. Analysis of the synovial cell infiltrate in early rheumatoid synovial tissue in relation to local disease activity. Arthritis Rheum 1997; 40:217-225.

55. Baeten D., Demetter P., Cuvelier C., et al. Comparative study of the synovial histology in rheumatoid arthritis, spondyloarthropathy, and osteoarthritis: influence of disease duration and activity. Ann Rheum Dis 2000; 59:945-953.

56. Reece R.J., Canete J.D., Parsons W.J., et al. Distinct vascular patterns of early synovitis in psoriatic, reactive, and rheumatoid arthritis. Arthritis Rheum 1999; 42:1481-1484.

57. Hitchon C.A., El-Gabalawy H.S. The histopathology of early synovitis. Clin Exp Rheumatol 2003; 21(Suppl31):S28-S36.
58. Lim G.Y., Im S.A., Jung W.S., et al. Evaluation of joint effusion in rabbits by color Doppler, power Doppler and contrast-enhanced power Doppler ultrasonography. J Clin Ultrasound 2005; 33:333338.

59. Jiang Y., Qiu L., Zhang L., et al. Noninvasive quantitative assessment of synovial pannus angio- genesis by contrastenhanced gray-scale sonography in antigen-induced arthritis in rabbits. Acad Radiol 2011; 18:359-368.

60. Qiu L., Jiang Y., Luo Y., et al. Antigen-induced arthritis in rabbits: a comparative study between high-resolution ultrasound and contrast-enhanced ultrasound and pathologic findings. Rheumatol Int 2012 Jun; 32(6):1569-1580.

61. Dietrich C.F., Averkiou M.A., Correas J.M., et al. An EFSUMB Introduction into Dynamic Contrast- Enhanced Ultrasound (DCEUS) for Quantification of Tumour Perfusion. Ultraschall in Med 2012; 33:344-351.

62. Klauser A.S., Franz M., Bellmann Weiler R., et al. Contrastenhanced ultrasonography for the detection of joint vascularity in arthritis-subjective grading versus computer-aided objective quantification. Ultraschall Med 2011 Dec; 32 Suppl 2:E31-37.

63. Salaffi F., Carotti M., Manganelli P., et al. Contrast-enhanced power Doppler sonography of knee synovitis in rheumatoid arthritis: assessment of therapeutic response. Clin Rheumatol 2004 Aug; 23(4):285-290

64. Schueller-Weidekamm C., Krestan C., Schueller G., et al. Power Doppler sonography and pulse-inversion harmonic imaging in evaluation of rheumatoid arthritis synovitis. Am J Roentgenol 2007 Feb; 188(2):504-508. 